\title{
Some Fixed Point Theorems of Integral Type Contraction in Cone b-metric Spaces
}

\author{
Rahim Shah*, Akbar Zada, Ishfaq Khan \\ Department of Mathematics, University of Peshawar, Peshawar, Pakistan \\ *Corresponding author: safeer_rahim@yahoo.com \\ Received August 09, 2015; Revised December 23, 2015; Accepted December 30, 2015
}

\begin{abstract}
In the present paper, we introduces the concept of integral type contraction with respect to cone b-metric space. Also we proved some fixed point results of integral type contractive mapping in cone b-metric space. We give an example to support our main result.
\end{abstract}

Keywords: cone b-metric space, fixed point, integral type contractive mapping

Cite This Article: Rahim Shah, Akbar Zada, and Ishfaq Khan, "Some Fixed Point Theorems of Integral Type Contraction in Cone b-metric Spaces." Turkish Journal of Analysis and Number Theory, vol. 3, no. 6 (2015): 165169. doi: 10.12691/tjant-3-6-5.

\section{Introduction}

The study of fixed point theory plays an important role in applications of many branches of mathematics. Finding a fixed point of contractive mappings becomes the center of strong research activity. There are some researchers who have worked about the fixed point of contractive mappings see [4,11]. In 1922, Banach [4] presented an important result regarding a contraction mapping, known as the Banach contraction principle. Bakhtin in [3] introduced the concept of b-metric spaces as a generalization of metric spaces. He proved the contraction mapping principle in b-metric spaces that generalized the famous Banach contraction principle in metric spaces. The concept of cone metric space was presented by Haung and Zhang [15] in 2007. They replace an ordered Banach space for the real numbers and proved some fixed point theorems of contractive mappings in cone metric space. Hussain and Shah give the concept of cone b-metric space as a generalization of b-metric space and cone metric space in [16]. Also they improved some recent results about KKM mappings in cone b-metric spaces.

In 2002, Branciari [8] introduced the notion of integral type contractive mappings in complete metric spaces and study the existence of fixed points for mappings which are defined on complete metric space satisfying integral type contraction. Recently F. Khojasteh et al. [19], presented the concept of integral type contraction in cone metric spaces and proved some fixed point theorems in such spaces. Many researchers studies various contractions and a lot of fixed point theorems are proved in different spaces; see $[1-7,9,10,11,12,13,17,18,20]$.

In the main section of this paper we presented some fixed point theorems of Integral type contractive mappings in setting of cone b-metric spaces. Moreover, we present suitable example that support our main result.

\section{Preliminaries}

The following definitions and results will be needed in this paper.

Definition 2.1 [15] Let $\mathbb{G}$ be a real Banach space and $\mathbb{K}$ be a subset of $\mathbb{G}$. Then $\mathbb{K}$ is called cone if and only if:

(i) $\mathbb{K}$ is closed, nonempty and $\mathbb{K} \neq\{0\}$;

(ii) $c p+d q \in \mathbb{K}$ for all $p, q \in \mathbb{K}$ where $c, d$ are nonnegative real numbers;

(iii) $\mathbb{K} \cap(-\mathbb{K})=\{0\}$.

Definition 2.2 [15] Suppose $\mathbb{K}$ be a cone in real Banach space $\mathbb{G}$, we define a partial ordering $\leq$ with respect to $\mathbb{K}$ by $p \leq q \Leftrightarrow q-p \in \mathbb{K}$. We shall write $p<q$ to indicate that $p \leq q$ but $p \neq q$, while $p \ll q$ will stand for $q-p \in \operatorname{int} \mathbb{K}$.

Definition 2.3 [15] The cone $\mathbb{K}$ is called normal if there is number $K>0$ such that for all $p, q \in \mathbb{G}, 0 \leq p \leq q$ implies $\|p\| \leq K\|q\|$.

The least positive number $K$ satisfying the above inequality is called the normal constant of cone.

Throughout this paper we always suppose that $\mathbb{G}$ is a real Banach space, $\mathbb{K}$ is a cone in $\mathbb{G}$ with int $\mathbb{K} \neq \varnothing$ and $\leq$ is partial ordering w.r.t cone.

Definition 2.4 [15] Let $Y$ be a non-empty set. Suppose that the mapping $d: Y \times Y \rightarrow \mathbb{K}$ satisfies:

(d1) $0 \leq d(u, v)$ for all $u, v \in Y$ with $u \neq v$;

(d2) $d(u, v)=0$ if and only if $u=v$;

(d3) $d(u, v)=d(v, u)$ for all $u, v \in Y$;

(d4) $d(u, v) \leq d(u, w)+d(w, v)$ for all $u, v, w \in Y$.

Then $d$ is called a cone metric on $Y$ and $(Y, d)$ is called $a$ cone metric space. 
Example 2.5 [15] Suppose $\mathbb{G}=R^{2}, \mathbb{K}=\{(u, v) \in \mathbb{G} \mid u, v \geq 0\}$ $\subset R^{2}, \quad Y=R$ and $d: Y \times Y \rightarrow \mathbb{G}$ such that $d(u, v)$ $=(|u-v|, \alpha|u-v|)$, where $\alpha \geq 0$ is a constant. Then $(Y, d)$ is cone metric space.

Definition 2.6 [16] Let $Y$ be a non-empty set and $s \geq 1$ be a given real number. A mapping $d: Y \times Y \rightarrow \mathbb{K}$ is said to be cone b-metric if and only if, for all $u, v, w$ in $Y$, the following conditions are satisfied:

(i) $0 \leq d(u, v)$ for all $u, v \in Y$ with $u \neq v$;

(ii) $d(u, v)=0$ if and only if $u=v$;

(iii) $d(u, v)=d(v, u)$ for all $u, v \in Y$;

(iv) $d(u, v) \leq s[d(u, w)+d(w, v)]$ for all $u, v, w \in Y$.

Then $d$ is called a cone $b$-metric on $Y$ and $(Y, d)$ is called $a$ cone b-metric space.

Example 2.7 [14] Let $\mathbb{G}=R^{2}, \mathbb{K}=\{(u, v) \in \mathbb{G} \mid u, v \geq 0\} \subset R^{2}$, $d: Y \times Y \rightarrow \mathbb{K}$ such that $d(u, v)=\left(|u-v|^{p}, \alpha|u-v|^{p}\right)$, where $\alpha \geq 0$ and $p>1$ are constants. Then $(Y, d)$ is cone b-metric space.

Lemma 2.8 [15] Let $(Y, d)$ be a cone metric space and $\mathbb{K}$ a normal cone with normal constant $K$. Let $\left\{u_{n}\right\}_{n \in N}$ be a sequence in $Y$. Then $\left\{u_{n}\right\}_{n \in N}$ converges to $u$ if and only if

$$
\lim _{n \rightarrow \infty} d\left(u_{n}, u\right)=0
$$

Lemma 2.9 [15] Let $(Y, d)$ be a cone metric space and $\mathbb{K}$ a normal cone with normal constant $K$. Let $\left\{u_{n}\right\}_{n \in N}$ be a sequence in $Y$. Then $\left\{u_{n}\right\}_{n \in N}$ is a Cauchy sequence if and only if

$$
\lim _{m, n \rightarrow \infty} d\left(u_{m}, u_{n}\right)=0
$$

Lemma 2.10 [15] Let $(Y, d)$ be a cone metric space and $\left\{u_{n}\right\}_{n \in N}$ a sequence in $Y$. If $\left\{u_{n}\right\}_{n \in N}$ is convergent, then it is a Cauchy sequence.

Lemma 2.11 [15] Let $(Y, d)$ be a cone metric space and $\mathbb{K}$ be a normal cone with normal constant $K$. Let $\left\{u_{n}\right\}$ and $\left\{v_{n}\right\}$ be two sequences in $Y$ and $u_{n} \rightarrow u, v_{n} \rightarrow v$ as $n \rightarrow \infty$. Then

$$
d\left(u_{n}, v_{n}\right) \rightarrow d(u, v) \text { as } n \rightarrow \infty .
$$

In 2002, Branciari in [8] introduced a general contractive condition of integral type as follows.

Theorem 2.12 [8] Let $(Y, d)$ be a complete metric space, $\alpha \in(0,1)$, and $f: Y \rightarrow Y$ is a mapping such that for all $x, y \in Y$,

$$
\int_{0}^{d(f(x), f(y))} \phi(t) d t \leq \alpha \int_{0}^{d(x, y)} \phi(t) d t
$$

where $\phi:[0,+\infty) \rightarrow[0,+\infty)$ is nonnegative and Lebesgueintegrable mapping which is summable (i.e., with finite integral) on each compact subset of $[0,+\infty)$ such that for each $\epsilon>0, \int_{0}^{\epsilon} \phi(t) d t>0$, then $f$ has a unique fixed point $a \in Y$, such that for each $x \in Y, \lim _{n \rightarrow \infty} f^{n}(x)=a$.

In [19], Khojasteh et al. defined new concept of integral with respect to a cone and introduce the Branciaris result in cone metric spaces. We recall their idea so that the paper will be self contained.

Definition 2.13 Suppose that $\mathbb{K}$ is a normal cone in $\mathbb{G}$. Let $a, b \in E$ and $a<b$. We define

$[a, b]:=\{x \in \mathbb{G}: x=t b+(1-t) a$, for some $t \in[0,1]\}$,

$[a, b):=\{x \in \mathbb{G}: x=t b+(1-t) a$, for some $t \in[0,1)\}$.

Definition 2.14 The set $\left\{a=x_{0}, x_{1}, \ldots, x_{n}=b\right\}$ is called $a$ partition for $[a, b]$ if and only if the sets $\left\{\left[x_{i-1}, x_{)}\right]\right\}_{i=1}^{n}$ are pairwise disjoint and $[a, b]=\left\{\bigcup_{i=1}^{n}\left[x_{i-1}, x_{)}\right)\right\} \cup\{b\}$.

Definition 2.15 For each partition $P$ of $[a, b]$ and each increasing function $\phi:[a, b] \rightarrow \mathbb{K}$, we define cone lower summation and cone upper summation as

$$
\begin{gathered}
L_{n}^{\text {Con }}(\phi, P):=\sum_{i=0}^{n-1} \phi\left(x_{i}\right)\left\|x_{i}-x_{i+1}\right\| \\
U_{n}^{\text {Con }}(\phi, P):=\sum_{i=0}^{n-1} \phi\left(x_{i+1}\right)\left\|x_{i}-x_{i+1}\right\|
\end{gathered}
$$

respectively.

Definition 2.16 Suppose that $\mathbb{K}$ is a normal cone in $\mathbb{G}$. $\phi:[a, b] \rightarrow \mathbb{K}$ is called an integrable function on $[a, b]$ with respect to cone $\mathbb{K}$ or to simplicity, Cone integrable function, if and only if for all partition $P$ of $[a, b]$

$$
\lim _{n \rightarrow \infty} L_{n}^{\text {Con }}(\phi, P)=S^{\text {Con }}=\lim _{n \rightarrow \infty} U_{n}^{\text {Con }}(\phi, P)
$$

where $S^{\text {Con }}$ must be unique.

We show the common value $S^{\text {Con }}$ by

$$
\int_{a}^{b} \phi(x) d_{\mathbb{K}}(x) \text { simply by } \int_{a}^{b} \phi(x) d_{\mathbb{K}} \text {. }
$$

Let $\mathcal{L}^{1}([a, b], \mathbb{K})$ denotes the set of all cone integreble functions.

Lemma 2.17 [19] Let $f, g \in \mathcal{L}^{1}([a, b], \mathbb{K})$. The following two statements hold.

- (1) If $[a, b] \subset[a, c]$, then $\int_{a}^{b} f d_{\mathbb{K}} \leq \int_{a}^{c} f d_{\mathbb{K}}$, for $f \in \mathcal{L}^{1}([a, b], \mathbb{K})$.

- (2) $\int_{a}^{b}(\alpha f+\beta g) d_{\mathbb{K}}=\alpha \int_{a}^{b} f d_{\mathbb{K}}+\beta \int_{a}^{b} g d_{\mathbb{K}}$ for $\alpha, \beta \in \mathbb{R}$.

Definition 2.18 [19] The function $\varphi: \mathbb{K} \rightarrow \mathbb{K}$ is called subadditive cone integrable function if and only if for all $c, d \in \mathbb{K}$ 


$$
\int_{0}^{c+d} \varphi d_{\mathbb{K}} \leq \int_{0}^{c} \varphi d_{\mathbb{K}}+\int_{a}^{d} \varphi d_{\mathbb{K}}
$$

Example 2.19 [19] Let $\mathbb{G}=Y=R, \quad d(u, v)=|u-v|$, $\mathbb{K}=[0,+\infty)$, and $\varphi(t)=\frac{1}{t+1}$ for all $t>0$ then for all $c, d \in \mathbb{K}$,

$$
\begin{aligned}
& \int_{0}^{c+d} \frac{d t}{t+1}=\ln (c+d+1), \\
& \int_{0}^{c} \frac{d t}{t+1}=\ln (c+1), \\
& \text { and } \int_{0}^{d} \frac{d t}{t+1}=\ln (d+1) .
\end{aligned}
$$

Since $c d \geq 0$ thus $c+d+1 \leq c+d+1+c d \leq(c+1)(d+1)$, therefore

$$
\ln (c+d+1) \leq \ln ((c+1)(d+1))=\ln (c+1)+\ln (d+1) .
$$

Which shows that $\varphi$ is a subadditive cone integrable function.

Theorem 2.20 [19] Let $(Y, d)$ be a complete regular cone metric space and $H$ be a mapping on $Y$. Suppose that there exist a function $\theta$ from $\mathbb{K}$ into itself which satisfies: (i) $\theta(0)=0$ and $\theta(t) \gg 0$ for all $t \gg 0$.

(ii) The function $\theta$ is nondecreasing and continuous. Moreover, its inverse is also continuous.

(iii) For all $0 \neq \epsilon \in \mathbb{K}$, there exist $\delta \gg 0$ such that for all $a, b \in Y$

$$
\theta(d(a, b))<\epsilon+\delta \text { implies } \theta(d(H a, H b))<\epsilon .
$$

(iv) For all $a ; b 2 Y$

$$
\theta(a, b) \leq \theta(a)+\theta(b) .
$$

Then the function $H$ has a unique fixed point.

Remark 2.21 [19] If $\varphi: \mathbb{K} \rightarrow \mathbb{K}$ is a non-vanishing map and a sub-additive cone integrable on each $[a, b] \subset \mathbb{K}$ such that for each $\epsilon \gg 0, \quad \int_{0}^{\epsilon} \varphi d_{\mathbb{K}} \gg 0$ and $\theta(x)=\int_{0}^{x} \varphi d_{\mathbb{K}}$ must have the continuous inverse, then $\theta$ is satisfies in all conditions in Theorem 2.20.

\section{Main Results}

In this section we presented some fixed point results in cone b-metric space by using integral type contractive mappings. Our main result is stated as follows.

Theorem 3.1 Let $(Y, d)$ be a complete cone b-metric space with $s \geq 1$ and $\mathbb{K}$ be a normal cone. Let the mapping $\varphi: \mathbb{K} \rightarrow \mathbb{K}$ is a nonvanishing map and subadditive cone integrable $R$ on each $[a, b] \subset \mathbb{K}$ such that for each $\epsilon \gg 0, \quad \int_{0}^{\epsilon} \varphi d_{\mathbb{K}} \gg 0$ must have the continuous inverse. If $H: Y \rightarrow Y$ is a map such that, for all $u, v \in Y$

$$
\int_{0}^{d(H u, H v)} \varphi d_{\mathbb{K}} \leq \lambda \int_{0}^{d(u, v)} \varphi d_{\mathbb{K}}
$$

where $\lambda \in(0,1)$ is a constant. Then $H$ has a unique fixed point in $Y$.

Proof. Let $u_{0} \in Y$. Choose $u_{n+1}=H u_{n}$.

We have

$$
\begin{aligned}
\int_{0}^{d\left(u_{n+1}, u_{n}\right)} \varphi d_{\mathbb{K}} & =\int_{0}^{d\left(H_{u_{n}}, H_{u_{n-1}}\right)} \varphi d_{\mathbb{K}} \\
& \leq \lambda \int_{0}^{d\left(u_{n}, u_{n-1}\right)} \varphi d_{\mathbb{K}} \\
& \cdot \\
& \cdot \\
& \cdot \\
& \leq \lambda^{n} \int_{0}^{d\left(u_{1}, u_{0}\right)} \varphi d_{\mathbb{K}} .
\end{aligned}
$$

Since $\lambda \in(0,1)$ thus

$$
\lim _{n \rightarrow \infty} \int_{0}^{d\left(u_{n+1}, u_{n}\right)} \varphi d_{\mathbb{K}}=0 .
$$

If $\lim _{n \rightarrow \infty} d\left(u_{n+1}, u_{n}\right) \neq 0$ then $\lim _{n \rightarrow \infty} \int_{0}^{d\left(u_{n+1}, u_{n}\right)} \varphi d_{\mathbb{K}} \neq 0$ and this becomes contradiction, so

$$
\lim _{n \rightarrow \infty} d\left(u_{n+1}, u_{n}\right)=0 .
$$

Next we will show that $\left\{u_{n}\right\}$ is a Cauchy sequence. So, for any $m \geq 1, p \geq 1$

$$
\begin{aligned}
& \lim _{n \rightarrow \infty} d\left(u_{m+p}, u_{m}\right)=0 \text { as } m \rightarrow \infty . \\
& \int_{0}^{d\left(u_{m+p}, u_{m}\right)} \varphi d_{\mathbb{K}} \\
& \leq \int_{0}^{s\left[d\left(u_{m+p}, u_{m+p-1}\right)+d\left(u_{m+p-1}, u_{m}\right)\right]} \varphi d_{\mathbb{K}} \\
& =\int_{0}^{s d\left(u_{m+p}, u_{m+p-1}\right)} \varphi d_{\mathbb{K}}+\int_{0}^{s d\left(u_{m+p-1}, u_{m}\right)} \varphi d_{\mathbb{K}} \\
& \leq \int_{0}^{s d\left(u_{m+p}, u_{m+p-1}\right)} \varphi d_{\mathbb{K}} \\
& +\int_{0}^{s^{2}}\left[d\left(u_{m+p-1}, u_{m+p-2}\right)+d\left(u_{m+p-2}, u_{m}\right)\right] \varphi d_{\mathbb{K}} \\
& =\int_{0}^{s d\left(u_{m+p}, u_{m+p-1}\right)} \varphi d_{\mathbb{K}} \\
& +\int_{0}^{s^{2} d\left(u_{m+p-1}, u_{m+p-2}\right)} \varphi d_{\mathbb{K}}+\int_{0}^{s^{2} d\left(u_{m+p-2}, u_{m}\right)} \varphi d_{\mathbb{K}} \\
& \leq \int_{0}^{s d\left(u_{m+p}, u_{m+p-1}\right)} \varphi d_{\mathbb{K}}+\int_{0}^{s^{2} d\left(u_{m+p-1}, u_{m+p-2}\right)} \varphi d_{\mathbb{K}} \\
& +\int_{0}^{s^{3} d\left(u_{m+p-2}, u_{m+p-3}\right)} \varphi d_{\mathbb{K}}+\ldots \\
& +\int_{0}^{s^{p-1} d\left(u_{m+2}, u_{m+1}\right)} \varphi d_{\mathbb{K}}+\int_{0}^{s^{p-1} d\left(u_{m+1}, u_{m}\right)} \varphi d_{\mathbb{K}} \\
& \leq \lambda^{m+p-1} \int_{0}^{s d\left(u_{1}, u_{0}\right)} \varphi d_{\mathbb{K}}+\lambda^{m+p-2} \int_{0}^{s^{2} d\left(u_{1}, u_{0}\right)} \varphi d_{\mathbb{K}} \\
& +\lambda^{m+p-3} \int_{0}^{s^{3} d\left(u_{1}, u_{0}\right)} \varphi d_{\mathbb{K}}+\ldots
\end{aligned}
$$

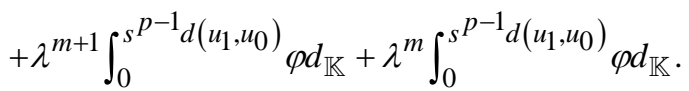


Since $\lambda \in(0,1)$, so $\lim _{m \rightarrow \infty} \int_{0}^{d\left(u_{m+p}, u_{m}\right)} \varphi d_{\mathbb{K}}=0$. By a property of function $\varphi$, we obtain $\lim _{m \rightarrow \infty} d\left(u_{m+p}, u_{m}\right)=0$. This means that $\left\{u_{n}\right\}$ is Cauchy sequence. Since $(Y, d)$ is complete cone b-metric space, their exist $u^{*} \in Y$, such that $u_{n} \rightarrow u^{*}$ as $n \rightarrow \infty$. Since

$$
\begin{aligned}
& \int_{0}^{d\left(H u^{*}, u^{*}\right)} \varphi d_{\mathbb{K}} \leq \int_{0}^{s\left[d\left(H u^{*}, H u_{n}\right)+d\left(H u_{n}, u^{*}\right)\right]} \varphi d_{\mathbb{K}} \\
& \leq \int_{0}^{s d}\left(H u^{*}, H u_{n}\right) \varphi d_{\mathbb{K}}+\int_{0}^{s d}\left(H u_{n}, u^{*}\right) \varphi d_{\mathbb{K}} \\
& \leq \lambda \int_{0}^{s d}\left(u^{*}, u_{n}\right) \varphi d_{\mathbb{K}}+\int_{0}^{s d}\left(u_{n+1}, u^{*}\right) \varphi d_{\mathbb{K}} \rightarrow 0 .
\end{aligned}
$$

By using Lemma 2.8. Hence $d\left(H u^{*}, u^{*}\right)=0$. This implies $H u^{*}=u^{*}$. So $u^{*}$ is a fixed point of $H$. For uniqueness, now if $v^{*}$ is another fixed point of $H$, then

$$
\int_{0}^{d\left(u^{*}, v^{*}\right)} \varphi d_{\mathbb{K}}=\int_{0}^{d\left(H u^{*}, H v^{*}\right)} \varphi d_{\mathbb{K}} \leq \lambda \int_{0}^{d\left(u^{*}, v^{*}\right)} \varphi d_{\mathbb{K}}
$$

which is contradiction. Thus $H$ have a unique fixed point $u^{*} \in Y$.

Corollary 3.2 Let $(Y, d)$ be a complete cone b-metric space with $s \geq 1$ and $\mathbb{K}$ be a normal cone. Let the mapping $\varphi: \mathbb{K} \rightarrow \mathbb{K}$ is a nonvanishing map and subadditive cone integrable $R$ on each $[a, b] \subset \mathbb{K}$ such that for each $\epsilon \gg 0, \quad \int_{0}^{\epsilon} \varphi d_{\mathbb{K}} \gg 0$ must have the continuous inverse. If $H: Y \rightarrow Y$ is a map such that, for all $u, v \in X$

$$
\int_{0}^{d\left(H^{n} u, H^{n} v\right)} \varphi d_{\mathbb{K}} \leq \lambda \int_{0}^{d(u, v)} \varphi d_{\mathbb{K}}
$$

where $\lambda \in(0,1)$ is a constant. Then $H$ has a unique fixed point in $Y$.

Proof. From Theorem 3.1, $H^{n}$ has a unique fixed point $u^{*}$.

But $H^{n}\left(H u^{*}\right)=H\left(H^{n} u^{*}\right)=H u^{*}$, so $H u^{*}$ is also a fixed point of $H^{n}$. Hence $H u^{*}=u^{*}$, this means that $u^{*}$ is a fixed point of $H$. Thus the fixed point of $H^{n}$ is also a fixed point of $H$. Hence the fixed point of $H$ is unique.

Theorem 3.3 Let $(Y, d)$ be a complete cone b-metric space with $s=1$ and $\mathbb{K}$ be a normal cone. Let the mapping $\varphi: \mathbb{K} \rightarrow \mathbb{K}$ is a nonvanishing map Rand subadditive cone integrable on each $[a, b] \subset \mathbb{K}$ such that for each $\epsilon \gg 0, \quad \int_{0}^{\epsilon} \varphi d_{\mathbb{K}} \gg 0$ must have the continuous inverse. If $H: Y \rightarrow Y$ is a map such that, for all $u, v \in X$

$$
\int_{0}^{d(H u, H v)} \varphi d_{\mathbb{K}} \leq \lambda \int_{0}^{[d(u, H u)+d(v, H v)]} \varphi d_{\mathbb{K}}
$$

where $\lambda \in\left(0, \frac{1}{2}\right)$ is a constant. Then $H$ has a unique fixed point in $Y$.

Proof. Let $u_{0} \in Y$. Choose $u_{n+1}=H u_{n}$. We have

$$
\begin{aligned}
& \int_{0}^{d\left(u_{n+1}, u_{n}\right)} \varphi d_{\mathbb{K}}=\int_{0}^{d\left(H u_{n}, H u_{n-1}\right)} \varphi d_{\mathbb{K}} \\
& \leq \lambda \int_{0}^{d\left(u_{n}, H u_{n}\right)} \varphi d_{\mathbb{K}}+\lambda \int_{0}^{d\left(u_{n-1}, H u_{n-1}\right)} \varphi d_{\mathbb{K}} \\
& \leq \frac{\lambda}{1-\lambda} \int_{0}^{d\left(u_{n}, u_{n-1}\right)} \varphi d_{\mathbb{K}} \\
& \leq \alpha \int_{0}^{d\left(u_{n}, u_{n-1}\right)} \varphi d_{\mathbb{K}} \text { where } \alpha=\frac{\lambda}{1-\lambda} \\
& \leq \alpha^{n} \int_{0}^{d\left(u_{1}, u_{0}\right)} \varphi d_{\mathbb{K}} \cdot
\end{aligned}
$$

Next we will show that $\left\{u_{n}\right\}$ is a Cauchy sequence. So, for any $m \geq 1, p \geq 1$

$$
\lim _{m \rightarrow \infty} d\left(u_{m+p}, u_{m}\right)=0 \text { as } m \rightarrow \infty \text {. }
$$

$$
\begin{aligned}
& \int_{0}^{d\left(u_{m+p}, u_{m}\right)} \varphi d_{\mathbb{K}} \\
& \leq \int_{0}^{s}\left[d\left(u_{m+p}, u_{m+p-1}\right)+d\left(u_{m+p-1}, u_{m}\right)\right] \varphi d_{\mathbb{K}} \\
& =\int_{0}^{s d\left(u_{m+p}, u_{m+p-1}\right)} \varphi d_{\mathbb{K}}+\int_{0}^{s d\left(u_{m+p-1}, u_{m}\right)} \varphi d_{\mathbb{K}} \\
& \leq \int_{0}^{s d\left(u_{m+p}, u_{m+p-1}\right)} \varphi d_{\mathbb{K}} \\
& +\int_{0}^{s^{2}}\left[d\left(u_{m+p-1}, u_{m+p-2}\right)+d\left(u_{m+p-2}, u_{m}\right)\right] \varphi d_{\mathbb{K}} \\
& =\int_{0}^{s d\left(u_{m+p}, u_{m+p-1}\right)} \varphi d_{\mathbb{K}} \\
& +\int_{0}^{s^{2} d\left(u_{m+p-1}, u_{m+p-2}\right)} \varphi d_{\mathbb{K}}+\int_{0}^{s^{2} d\left(u_{m+p-2}, u_{m}\right)} \varphi d_{\mathbb{K}} \\
& \leq \int_{0}^{s d\left(u_{m+p}, u_{m+p-1}\right)} \varphi d_{\mathbb{K}}+\int_{0}^{s^{2} d\left(u_{m+p-1}, u_{m+p-2}\right)} \varphi d_{\mathbb{K}} \\
& +\int_{0}^{s^{3} d\left(u_{m+p-2}, u_{m+p-3}\right)} \varphi d_{\mathbb{K}}+\ldots \\
& +\int_{0}^{s^{p-1} d\left(u_{m+2}, u_{m+1}\right)} \varphi d_{\mathbb{K}}+\int_{0}^{s^{p-1} d\left(u_{m+1}, u_{m}\right)} \varphi d_{\mathbb{K}} \\
& \leq \alpha^{m+p-1} \int_{0}^{s d\left(u_{1}, u_{0}\right)} \varphi d_{\mathbb{K}}+\alpha^{m+p-2} \int_{0}^{s^{2} d\left(u_{1}, u_{0}\right)} \varphi d_{\mathbb{K}} \\
& +\alpha^{m+p-3} \int_{0}^{s^{3} d\left(u_{1}, u_{0}\right)} \varphi d_{\mathbb{K}}+\ldots \\
& +\alpha^{m+1} \int_{0}^{s^{p-1} d\left(u_{1}, u_{0}\right)} \varphi d_{\mathbb{K}}+\alpha^{m} \int_{0}^{s^{p-1} d\left(u_{1}, u_{0}\right)} \varphi d_{\mathbb{K}} \text {. }
\end{aligned}
$$

So $\lim _{m \rightarrow \infty} \int_{0}^{d\left(u_{m+p}, u_{m}\right)} \varphi d_{\mathbb{K}}=0$. By a property of function $\varphi$, we obtain $\lim _{m \rightarrow \infty} d\left(u_{m+p}, u_{m}\right)=0$. This means that $\left\{u_{n}\right\}$ is Cauchy sequence. Since $(Y, d)$ is complete cone $b$-metric space, their exist $u^{*} \in Y$, such that $u_{n} \rightarrow u^{*}$ as $n \rightarrow \infty$. Since 


$$
\begin{aligned}
& \int_{0}^{d\left(u^{*}, H u^{*}\right)} \varphi d_{\mathbb{K}} \\
& \leq \int_{0}^{s\left[d\left(u^{*}, u_{n}\right)+d\left(u_{n}, u_{n+1}\right)+d\left(u_{n+1}, H u^{*}\right)\right]} \varphi d_{\mathbb{K}} \\
& \leq \int_{0}^{s\left[d\left(u^{*}, u_{n}\right)+d\left(u_{n}, u_{n+1}\right)+d\left(H u_{n}, H u^{*}\right)\right]} \varphi d_{\mathbb{K}} \\
& \leq \int_{0}^{s d\left(u^{*}, u_{n}\right)} \varphi d_{\mathbb{K}}+\int_{0}^{s d\left(u_{n}, u_{n+1}\right)} \varphi d_{\mathbb{K}} \\
& +\lambda \int_{0}^{s\left[d\left(u_{n}, H u_{n}\right)+d\left(u^{*}, H u^{*}\right)\right]} \varphi d_{\mathbb{K}} \\
& \leq \frac{1}{1-\lambda} \int_{0}^{s d\left(u^{*}, u_{n}\right)} \varphi d_{\mathbb{K}}+(1+\lambda) \frac{\lambda^{n}}{1-\lambda} \int_{0}^{s d\left(u_{0}, u_{1}\right)} \varphi d_{\mathbb{K}} \rightarrow 0 .
\end{aligned}
$$

Hence $d\left(u^{*}, H u^{*}\right)=0$. This implies $u^{*}=H u^{*}$. So $u^{*}$ is a fixed point of $H$. For uniqueness, now if $v^{*}$ is another fixed point of $H$, then

$$
\begin{aligned}
& \int_{0}^{d\left(u^{*}, v^{*}\right)} \varphi d_{\mathbb{K}}=\int_{0}^{d\left(H u^{*}, H v^{*}\right)} \varphi d_{\mathbb{K}} \\
& \leq \lambda \int_{0}^{\left[d\left(u^{*}, H u^{*}\right)+d\left(v^{*}, H v^{*}\right)\right]} \varphi d_{\mathbb{K}} \\
& \leq \lambda \int_{0}^{\left[d\left(u^{*}, u^{*}\right)+d\left(v^{*}, v\right)\right]} \varphi d_{\mathbb{K}} \cdot
\end{aligned}
$$

We have $d\left(u^{*}, v^{*}\right)=0$. Hence $u^{*}=v^{*}$. Thus $u^{*}$ is the unique fixed point of $H$.

Example 3.4 Let $Y=[0,1], \mathbb{G}=\mathbb{R}^{2}$ and $p>1$ be a constant. Take $\mathbb{K}=\{(u, v) \in \mathbb{G}: u, v \geq 0\}$. We define $d: Y \times Y \rightarrow \mathbb{G}$ as

$$
d(u, v)=\left(|u-v|^{p},|u-v|^{p}\right) .
$$

Then $(Y, d)$ is complete cone b-metric space. Suppose $H: Y \times Y$ as

$H u=\frac{1}{2} u-\frac{1}{4} u^{2}$ for all $u \in Y$ and $\varphi(k)=1$ where $k \in \mathbb{R}$.

Then the condition of Theorem 3.1 holds, in fact

$$
\begin{aligned}
& \int_{0}^{d(H u, H v)} \varphi d_{\mathbb{K}}=\int_{0}^{\left(|u-v|^{p},|u-v|^{p}\right)} \varphi d_{\mathbb{K}} \\
& =\int_{0}^{\left(\left|\frac{1}{2}(u-v)-\frac{1}{4}(u-v)(u+v)\right|^{p},\left|\frac{1}{2}(u-v)-\frac{1}{4}(u-v)(u+v)\right|^{p}\right)} \varphi d_{\mathbb{K}} \\
& =\int_{0}^{\left(|u-v|^{p}\left|\frac{1}{2}-\frac{1}{4}(u+v)\right|^{p},|u-v|^{p}\left|\frac{1}{2}-\frac{1}{4}(u+v)\right|^{p}\right)} \varphi d_{\mathbb{K}} \\
& \leq \frac{1}{2^{p}} \int_{0}^{\left(|u-v|^{p},|u-v|^{p}\right)} \varphi d_{\mathbb{K}} \\
& \leq \frac{1}{2^{p}} \int_{0}^{d(u, v)} \varphi d_{\mathbb{K} \cdot} \cdot
\end{aligned}
$$

Here $0 \in Y$ is the unique fixed of $H$.

\section{References}

[1] A. Azam, M. Arshad, Kannan fixed point theorems on generalized metric spaces, J. Nonlinear Sci. Appl., vol. 1, 2008, pp. 45-48.

[2] A. Azam, M. Arshad, I. Beg, Banach contraction principle on cone rectengular metric spaces, Applicable Anal. Discrete Math., vol. 3, 2009, pp. 236-241.

[3] I. A. Bakhtin, The contraction mapping principle in almost metric spaces, Func. Anal. Gos. Ped. Inst. Unianowsk, vol. 30, 1989, pp. 26-37.

[4] S. Banach, Sur les operations dans les ensembles abstrait et leur application aux equations, integrals, Fundan. Math., vol. 3, 1922, pp. 133-181.

[5] M. Boriceanu, Strict fixed point theorems for multivalued operators in b-metric spaces, Intern. J. Modern Math., vol. 4(3), 2009, pp. 285-301.

[6] M. Boriceanu, M. Bota, A. Petrusel, Multivalued fractals in bmetric spaces, Cent. Eur. J. Math., vol. 8(2), 2010, pp. 367-377.

[7] M. Bota, A. Molnar, V. Csaba, On Ekeland's variational principal in b-metric spaces, Fixed Point Theory, vol. 12, 2011, pp. 21-28.

[8] A. Branciari, A xed point theorem for mappings satisfying a general contractive condition of integral type, International Journal of Mathematics and Mathematical Sciences, vol. 29, no. 9, 2002, pp. 531-536.

[9] S. Czerwik, Cotraction mapping in b-metric spaces, Acta. Math. Inform. Univ. Ostraviensis, vol. 1, 1993, pp. 5-11.

[10] P. Das, B.K. Lahiri, Fixed point of cotractive mappings in generalized metric space, Math. Slovaca., vol. 59, 2009, pp. 499501

[11] K. Deimling, Nonlinerar Functional Analysis, Springer, 1985.

[12] I.M. Erhan, E. Karapinar, T. Sekulic, Fixed points of $(\psi, \varphi)$ contractions on generalized metric space, Fixed Point Theory Appl., 2012, 220, (2012).

[13] R. George, B. Fisher, Some generalized results in cone b-metric space, Math. Moravica., vol. 17(2), 2013, pp. 39-50.

[14] H. H. Haung, S. Xu, Fixed point theorems of contractive mappings in cone b-metric spaces and applications, Fixed Point Theory Appl., 2012(220), 2012.

[15] L. G. Haung, X. Zhang, Cone metric space and _xed point theorems of contractive mappings, J. Math. Anal., Apal, vol. 332(2), 2007, pp. 1468-1476.

[16] N. Hussain, MH. Shah, KKM mapping in cone b-metric spaces, Comput. Math. Appl., vol.62, 2011, pp. $1677\{1684$.

[17] M. Jleli, B. Zhang, The Kannan's fixed point theorem in a cone rectengular metric space, J. Nonlinear Sci. Apal., vol. 2(3), 2009, pp. 161-167.

[18] Z. Kadelburg, S. Radenović, On generalized metric space, TWMS J. Pure. Appl. Math., vol. 5(1), 2014, pp. 03-13.

[19] F. Khojasteh, Z. Goodarzi, A. Razani, Some Fixed Point Theorems of Integral Type Contraction in Cone Metric Spaces, Fixed Point Theory Appl., vol. 2010, Article ID 189684, 13 pages, 2010.

[20] A. Zada, R. Shah, T. Li, Integral Type Contraction and Coupled Coincidence Fixed Point Theorems for Two Pairs in G-metric Spaces, Hacet. J. Math. Stat. 